\title{
Monoclonal Antibody-Drug Conjugate Therapy for the Patients with Colorectal Gancer
}

\author{
Toshio Takahashi, Toshiharu Yamaguchi, Kazuya \\ Kitamura, Akinori Noguchi and Mitsuyo Honda
}

The First Department of Surgery, Kyoto Prefectural University of Medicine, Kyoto 602

\begin{abstract}
Takahashi, T., Yamaguchi, T., Kitamura, K., Noguchi, A. and Honda, M. Monoclonal Antibody-Drug Conjugate Therapy for the Patients with Colorectal Cancer. Tohoku J. Exp. Med., 1992, 168 (2), 371-374_- Monoclonal antibody drug conjugate $\mathbf{A} 7$ was prepared from a mouse splenocyte immunized against human colon cancer. A7 reacted with 80 percent of colorectal cancer and pancreatic cancer. A7 was bound covalently to neocarzinostatin (NCS) to form A7-NCS. A7-NCS had strong cytotoxic activity in vivo and in vitro study. A total of 77 patients with colorectal cancer, including the patients with liver, lung and peritoneal metastasis, were treated with A7-NCS. There were some tumor reduction of liver metastasis on CT scan and pain relief. Follow up study of colorectal cancer patients treated with monoclonal antibody drug conjugate A7-NCS was carried out, with comparing to those treated conventional chemotherapy. Survival rate of the patients with postoperative liver metastasis treated with A7-NCS was slightly higher than that of the patients treated with conventional intraarterial infusion chemotherapy. There was no difference between the group treated with A7-NCS and that treated with conventional chemotherapy in the overall postoperative survival. Patients given a higher dose of the conjugate had a higher survival rate. There were no serious adverse effects in the patients given A7-NCS. Human anti-mouse antibody (HAMA) was detected in all A7-NCS treated patients. monoclonal antibody; neocarcinostatin; colorectal cancer; antibodydrug conjugate; cytotoxicity
\end{abstract}

One of major problem with cancer chemotherapy is the lack of tumor specificity and detrimental effect of the anticancer drug on normal cells. To overcome this problem, we have been engaged in this area of research since 1980 (Takahashi et al. 1980, 1988 ; Kotanagi et al. 1986 ; Kitamura et al. 1989). In 1985, we initiated clinical trials of the monoclonal antibody-drug conjugate A7-NCS, and obtained some good results such as pain relif and decrease of liver metastasis in size on CT scans. This paper describes briefly the results and follow up results of these trials of 77 patients with colorectal cancer, including postoperative metastases.

Address for reprints : Kawaramachi Hirokoji, Kamigyo-ku, Kyoto 602, Japan. 


\section{Materials and Methods}

Monoclonal antibody (A7). The monoclonal antibody, A7, (Kotanagi et al. 1986 ; Kitamura et al. 1989) from a mouse splenocyte immunized against human colon cancer was used in this series. The A7 was directed to an antigen of glycoprotein with a molecular weight of 40,000 and belonged to IgGl.

Anticancer drug. Neocarzinostatin (NCS), potent anticancer protein with high a molecular weight of 10,700 was used as the anticancer drug conjugating to the monoclonal antibody. NCS consists of apoprotein and chromophore.

Monoclonal antibody drug conjugate A7-NCS. A7 was bound covalently to the apoprotein portion of NCS. The conjugate A7-NCS contains $15 \mathrm{mg}$ of A7 per 1,000 units of NCS.

Patients receiving A\%-NCS. A total of 77 advanced cancer patients admitted to our Department of Surgery, Kyoto Prefectural University of Medicine from 1985 to 1990 were treated with A7-NCS ; Fifty five patients were advanced colorectal cancer and they underwent surgery for tumor removal. Nineteen patients were postoperative multiple liver metastasis, 2 were postoperative multiple lung metastasis and one was peritoneal metastasis after surgery for colorectal cancer.

Methods of administration. Thirty eight patients with primary colorectal cancers and 19 with postoperative liver metastases were given A7-NCS intraarterially by introducing a catheter inserted from the femoral or subclavian artery to the feeding artery of the tumor. Fourteen patients with primary colon cancers were given the conjugate intraoperatively from an artery proximal to the tumor. Two patients with lung metastases were given A7-NCS intravenously, and one patient with a peritoneal metastasis was given A7-NCS intraperitoneally. For 7 patients with postoperative liver metastases, we placed surgically a subcutaneous infuserport connected to a catheter inserted through the subclavian artery to the hepatic aretery for frequent injection of the conjugate. Sixty three patients were given the conjugate once, and 10 patients with postoperative liver or lung metastases were given it 3 to 5 times on successive days.

Dosage of $A 7-N C S$ administered. Five patients were injected with $15 \mathrm{mg}$ of $\mathrm{A} 7$ and 1,000 units of NCS. Twenty seven patients were given $30 \mathrm{mg}$ of A7 and 2,000 units of NCS. The other 35 patients received 45 to $90 \mathrm{mg}$ of A7 and 4,000 to 9,000 units of NCS. Six patients with postoperative liver metastases were given $225 \mathrm{mg}$ of A7 and 20,000 units of NCS by 5 consecutive injections from the port.

Additional treatment after A\%-NCS administration. All patients with primary colorectal cancers underwent surgery for resection of their cancers. Patients given A7-NCS did not receive any other chemotherapy.

Human antimouse antibody (HAMA). Blood serum was taken serially after the injection of A7-NCS. Antibodies against A7 were assayed by ELISA.

Follow up studies and evaluation. Patients were followed up for one to 5 years after injection of A7-NCS. The cumulative survival curves were generated by Kaplan Meiyer's method. Comparisons were made by a student's $t$-test between the patients treated with A7-NCS and those treated conventional treatments.

\section{RESULTS}

Of 19 patients with liver metastasis three patients showed clearly tumor reductions on CT scan and two patients had pain relief.

Follow up study of colorectal cancer patients treated with monoclonal antibody drug conjugate A7-NCS was carried out, being compared with those treated conventional chemotherapy. Survival rate of the patients with postoper- 
ative liver metastasis treated with A7-NCS was slightly higher than that of the those treated with intraarterial infusion chemotherapy and was almost the same rate as those treated with intra-arterial chemoembolization using multiple anticancer agents suspended in a lipid contrast medium. There was no difference between the group treated with A7-NCS and the group treated with conventional chemotherapy in the overall survival for the patients having undergone surgery.

Postoperative survival rate of the colorectal cancer patients with distant metastases to the liver, lung, peritoneum, and also to the lymph nodes (Dukes' D) showed the patients given A7-NCS exhibited slightly higher survival rate than those treated with conventional chemotherapy, but the difference was not statistically significant.

The survival curves were compared between patients given more than 4,000 units of NCS and less than 4,000 units. The patients given more than 4,000 units of NCS survived longer than those given less than 4,000 units of NCS. The patients given a higher dose of the conjugate had a higher survival rate. There were no serious adverse effects in the patients given A7-NCS. Human anti-mouse antibody (HAMA) was detected in all A7-NCS treated patients from seven to 500 days after the initial administration of A7-NCS. The patients tolerated well the repeated injections of a high dose of A7-NCS for five successive days. Humanmouse chimeric Fab of A7 was successfully prepared for the monoclonal antibody therapy in human.

\section{Discussion}

In our study, the conjugate produced some reductions in tumor size on CT scans and also produced pain relief in some patients with liver metastases.

To evaluate this treatment, it is necessary to make a randomized control study with 3 arms ; patients receiving no drug, NCS, and A7-NCS. However, we were not able to generate an accurate randomized control for this clinical trial because the treatment is not established as a common drug therapy. Accordingly, A7-NCS was given to a limited number of patients with considerably advanced cancers, and comparisons were made between patents receiving A7-NCS and those who underwent conventional treatments during the same 5 years.

Survival rate for patients with liver metastases treated with A7-NCS was higher than for patients with conventional chemotherapy and was approximately the same as that for patients receiving chemoembolization (Taniguchi et al. 1989) using multiple anticancer agents ( $5 \mathrm{FU}$, adriamycin and mitomycin) suspended in a lipid contrast medium. Although comparisons with patients given NCS alone were not obtained from this study, patients receives NCS alone do not experience survival periods of this length. Therefore, monoclonal antibody drug conjugate A7-NCS appears to be superior to conventional chemotherapy and allowed for a longer survival time for patients with liver metastasis from colorectal cancer. The A7-NCS, however, could not exceed chemoembolization which is the most 
powerful trargeting chemotherapy for liver metastasis up to the present.

Survival rate for the A7-NCS treated patients with distant metastases (Duke's D) was higher than that for the patients treated with conventional chemotherapy. Furthermore, it is interesting to note that the patients given a higher dose of the conjugate had a higher survival rate.

These results might suggest that the monoclonal antibody conjugate A7-NCS could contribute for a longer survival time than conventional chemotherapy.

There were no serious adverse side effects on patients given the conjugate, regardless of the administration route.

However, HAMA was detected in all patients examined.

Results obtained from this clinical observations suggest that cancer treatment using monoclonal antibody drug conjugates may be a promising improvement on conventional chemotherapy, although there are some problems to be resolved.

\section{References}

1) Kitamura, K., Takahashi, T., Yamaguchi, T., Yokota, T., Noguchi, T., Amagai, T. \& Imanishi, J. (1989) Immunochemical characterization of the antigen recognized by the murine monoclonal antibody A7 against human colorectal cancer. Tohoku $J$. Exp. Med., 157, 83-97.

2) Kotanagi, H., Takahashi, T. \& Masuko, T. (1986) A monoclonal antibody against human colon cancers. Tohoku J. Exp. Med., 148, 353-360.

3) Takahashi, T., Yamaguchi, T. \& Kohno, K. (1980) Selective chemotherapy of metastasis by drug carrying fat emulsion or tumor specific antibody. In : Metastasis, edited by K. Hellmann, P. Hilgard \& S. Eccles, Martinus Nijhoff, London, pp. 441-445.

4) Takahashi, T., Yamaguchi, T., Kitamura, K., Suzuyama, H., Honda, M., Yokota, T., Kotanagi, H., Takahashi, M. \& Hashimoto, Y. (1988) Clinical application of monoclonal antibody-drug conjugates for immunotargeting chemotherapy of colorectal carcinoma. Cancer, 61, 881-888.

5) Taniguchi, H., Takahashi, T., Yamaguchi, T. \& Sawai, K. (1989) Intraarterial infusion chemotherapy for metastatic liver tumor using multiple anti-cancer agents suspended in a lipid contrast medium. Cancer, 64, 2001-2006. 